\title{
Nonlinear Optical Properties of Carbon Nanotube Hybrids in Polymer Dispersions
}

\author{
Jun Wang \\ Chinese Academy of Sciences, \\ Kang-Shyang Liao \\ University of Houston \\ Daniel Früchtl \\ Trinity College Dublin
}

See next page for additional authors

Follow this and additional works at: https://arrow.tudublin.ie/nanolart

Part of the Atomic, Molecular and Optical Physics Commons

\section{Recommended Citation}

"Nonlinear optical properties of carbon nanotube hybrids in polymer dispersions", Jun Wang, et al. (2012) Materials Chemistry and Physics, 133, 992-997 (2012) doi:10.1016/j.matchemphys.2012.02.003

This Article is brought to you for free and open access by the NanoLab at ARROW@TU Dublin. It has been accepted for inclusion in Articles by an authorized administrator of ARROW@TU Dublin. For more information, please contact arrow.admin@tudublin.ie, aisling.coyne@tudublin.ie,gerard.connolly@tudublin.ie.

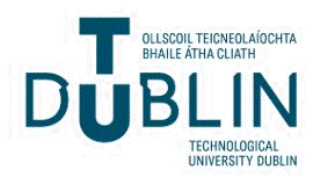




\section{Authors}

Jun Wang; Kang-Shyang Liao; Daniel Früchtl; Ying Tian; Aisling Gilchrist , T; Nigel Alley; Enrico Andreoli;

Brad Aitchison; Albert Nasibulin; Hugh Byrne; Esko I. Kauppinen; Long Zhang; Werner Blau; and Seamus Curran 


\section{Nonlinear Optical Properties of Carbon Nanotube Hybrids in Polymer Dispersions}

Jun Wang, ${ }^{1 *}$ Kang-Shyang Liao, ${ }^{2}$ Daniel Früchtl, ${ }^{3}$ Ying Tian, ${ }^{4}$ Aisling Gilchrist, ${ }^{3}$ Nigel J. Alley, ${ }^{2}$ Enrico Andreoli, ${ }^{2}$ Brad Aitchison, ${ }^{4}$ Albert G. Nasibulin, ${ }^{4}$ Hugh J. Byrne, ${ }^{5}$ Esko I. Kauppinen, ${ }^{4}$ Long Zhang, ${ }^{1 *}$ Werner J. Blau, ${ }^{3}$ and Seamus A. Curran ${ }^{2 *}$

${ }^{1}$ Key Laboratory of Materials for High-Power Laser, Shanghai Institute of Optics and Fine Mechanics, Chinese Academy of Sciences, Shanghai 201800, China

${ }^{2}$ Institute for NanoEnergy, Department of Physics, University of Houston, Houston, Texas 77204, USA

${ }^{3}$ School of Physics, Trinity College Dublin, Dublin 2, Ireland

${ }^{4}$ NanoMaterials Group, Department of Applied Physics and Center for New Materials, Aalto University, Puumiehenkuja 2, 00076, Finland

${ }^{5}$ Focas Research Institute, Dublin Institute of Technology, Dublin 8, Ireland

*Tel: +86 216991 4184, Fax: +86 216991 8932, E-mail: jwang@siom.ac.cn ; lzhang@siom.ac.cn; $\underline{\text { sacurran@uh.edu }}$ 


\section{Abstract}

A series of double-walled carbon nanotubes (DWNTs) and multi-walled nanotubes (MWNTs) functionalized with selected organic chromophores, fluorescein 5(6)-isothiocyanate (FITC), rhodamine B isothiocyanate (RITC) and fullerene $\left(\mathrm{C}_{60}\right)$ were synthesized by covalently linking these electron-donor groups to the metallic nanotubes. These versatile carbon nanotube composites show remarkable nonlinear optical (NLO) performance, due to a merged effect of the complementary NLO characteristics of the moieties of the composites. The hybrids were characterized by UV-Vis spectroscopy and Raman spectroscopy. Evidence suggests charge transfer species are formed between the chromophores and the nanotubes. The optical limiting performance of the DWNT, MWNT hybrids and carbon NanoBuds is found to be superior to that of single-walled nanotubes. Thermally induced light scattering from nanotube moieties is the primary mechanism dominating the NLO response. Reverse saturable absorption from fullerene moieties makes a significant contribution to the NLO response of fullerene containing hybrids and NanoBuds. Photoinduced charge transfer from the fullerenes or the organic chromophores (FITC and RITC) to nanotubes followed by absorption in the charge separated excited state may enhance the nonlinear absorption, thus an effective optical limiting response.

Keywords: carbon nanotube hybrids, optical limiting, Z-scan, nonlinear scattering. 


\section{Introduction}

Optical limiting is an important nonlinear optical (NLO) phenomenon, which can be utilized to protect delicate optical instruments, especially the human eye, from intense laser beams. Ideally an optical limiter should strongly attenuate intense, potentially dangerous laser beams, while exhibiting high transmittance for low intensity ambient light [1]. Up to now, people have found a large number of materials possessing optical limiting properties, e.g., phthalocyanines, porphyrins, fullerenes, carbon nanotubes, organic dyes, metal nanoclusters, etc. [2-6]. Along with the exploration of optical limiting materials, the physical mechanisms dominating the optical limiting have also been extensively investigated. In general, there are two main mechanisms for passive optical limiting: nonlinear absorption and nonlinear scattering. The former can be further divided into multi-photon absorption, reverse saturable absorption (RSA) and free-carrier absorption [1,4]. Inevitably, each mechanism or material has its intrinsic shortcomings, so that it alone cannot meet the idea requirements for laser protection. Nonlinearly absorbing materials generally exhibit fast response times in the picosecond (ps) regime, while the response spectral region is relatively narrow due to the limit of the resonance bandwidth. In contrast, the nonlinear scattering materials, e.g., carbon nanotubes, possess a broadband limiting effect but generally respond at best in the nanosecond (ns) regime $[4,5]$. A solution to improve the adaptability of optical limiting materials is to merge different functional materials, resulting in complementary temporal and spatial NLO characteristics and hence multi-functional limiting materials $[3,5]$. The large surface area of carbon nanotubes provides a platform to which many functional organic molecules or inorganic nanoparticles can be linked covalently or noncovalently, forming versatile composites [7-12]. 
Very recently, we reported the synthesis and enhanced optical limiting response of a double-walled carbon nanotube-fullerene nanohybrid [ 12 ]. In this work, double-walled nanotubes (DWNTs) and multi-walled nanotubes (MWNTs) were decorated with selected chromophores, specifically fluorescein 5(6)-isothiocyanate (FITC), rhodamine B isothiocyanate (RITC) and fullerene $\left(\mathrm{C}_{60}\right)$. By covalently linking these electron-donor groups to metallic nanotubes, the nanotube composites were expected to be promising donor-acceptor nano-systems upon photoexcitation. In 2007, Nasibulin et al. reported a novel carbon nanostructure NanoBuds, which covalently combine two carbon allotropes: fullerene and nanotubes [13]. Unlike the covalently linked organic molecules, the fullerene groups in NanoBuds directly grow on the outer surface of single-walled carbon nanotubes (SWNTs). We investigated the NLO properties of these nanohybrids and demonstrated their prominent optical limiting responses for nanosecond laser pulses in the visible regime. The results open up a gateway to develop multifunctional carbon nanocomposites for either NLO applications or, for instance, potential photovoltaic applications $[14,15]$. On the other hand, the donor-acceptor nanohybrids provide an opportunity to explore the contribution of charge transfer to NLO responses of the nanotube composites.

\section{Experimental section}

In addition to the DWNT- $\mathrm{C}_{60}$ reported previously [12], the nanocomposites in this work include DWNT-FITC, DWNT-RITC, MWNT-FITC, MWNT-RITC, MWNT-C 60 and NanoBuds. The detailed synthesis procedures of the DWNT nanohybrids are illustrated in Fig.1 and are described in the corresponding text. The MWNT composites are synthesized using analogous 
methods. The NanoBuds sample is synthesized using an aerosol reactor with ferrocene as the catalyst precursor and $\mathrm{CO}$ as the carbon source. $\mathrm{CO}_{2}$ is introduced into the reactor as an etching agent for the formation of the NanoBud structures [16].

Direct Amination of DWNTs. DWNTs (3 g) and polyethylenimine (PEI, $\left.\mathrm{M}_{\mathrm{n}} \sim 10,000\right)(15 \mathrm{~g})$ were mixed in $250 \mathrm{~mL}$ of N,N-dimethylformamide (DMF). Sonication for $20 \mathrm{~min}$ and stirring at $50{ }^{\circ} \mathrm{C}$ for $5 \mathrm{~d}$ formed the product, DWNT-PEI. The resulting suspension was filtered through a $0.20 \mu \mathrm{m}$ nylon membrane and the precipitate was washed with $1 \mathrm{M} \mathrm{HCl}, 1 \mathrm{M} \mathrm{NaOH}$, deionized water and methanol to remove any excess PEI. After drying, $5.5 \mathrm{~g}$ of the product was obtained.

Syntheses of DWNT-FITC or DWNT-RITC. DWNT-PEI (60 mg), Fluorescein 5(6)isothiocyanate (FITC, $60 \mathrm{mg}$ ), or Rhodamine B isothiocyanate (RITC, $60 \mathrm{mg}$ ) and triethylamine $(0.1 \mathrm{~mL})$ were mixed in $5 \mathrm{~mL}$ of DMF. Sonication for $2 \mathrm{~min}$ and stirring at $50{ }^{\circ} \mathrm{C}$ for $5 \mathrm{~d}$ formed the product, DWNT-FITC or DWNT-RITC. The resulting suspension was filtered through a 0.20 $\mu \mathrm{m}$ nylon membrane and the precipitate was washed with methanol and deionized water to remove any excess FITC or RITC. After drying, $60 \mathrm{mg}$ of the product was obtained.

Characterization. UV-vis spectra of the DWNT-FITC and the DWNT-RITC in water were recorded by an Ocean Optics HR2000+ high resolution spectrometer using a Mikropack PH2000-BAL Deuterium-Halogen light source. Raman spectra were collected using a Jobin Yvon Instruments S.A. LabRam 1B confocal Raman imaging microscope system. The source was a $514.5 \mathrm{~nm}$ Argon ion laser with maximum power of $50 \mathrm{~mW}$ at the sample. The Raman band of silicon $\left(520 \mathrm{~cm}^{-1}\right)$ was used to calibrate the spectrometer, with a resolution better than $1.5 \mathrm{~cm}^{-1}$ using an 1800 lines/mm grating. Raman samples were prepared by spin casting the sample suspension onto a silicon wafer. 


\section{Results and discussion}

We synthesized DWNT nanohybrids using commercially available reagents in only two steps. As shown in Fig. 1, DWNTs were first treated with an excess amount of polyethylenimine (PEI) in DMF at $50{ }^{\circ} \mathrm{C}$ to give DWNT-PEI by following a procedure described by Liao et al. [17 ]. The PEI functionalized DWNTs can be readily dispersed in common organic solvents and the free amines on PEI functionalized DWNTs can act as nucleophiles. In the next step, DWNT-PEI was reacted with an excess amount of FITC or RITC in DMF at $50{ }^{\circ} \mathrm{C}$ for 5 days. These chromophores covalently attach to the DWNT-PEI by thiourea bond formation. The successful formation of DWNT nanohybrids was first confirmed by UV-vis spectroscopy. As shown in Fig. 2, relative to the spectra of their corresponding chromophores (Fluorescein and Rhodamine B) dissolved in water, the spectra of DWNT-FITC and DWNT-RITC showed distinctive red-shifts from 440 to $530 \mathrm{~nm}$ and from 550 to $580 \mathrm{~nm}$, respectively. One possibility for these major redshifts can be the dyes effectively aggregate on the nanotube surfaces. To test this hypothesis, we also obtained the UV-vis spectra of Fluorescein and Rhodamine B dry film by drop casting. They clearly show that aggregation of the dye molecules cannot account for the major red-shifts we observed for the DWNT nanohybrids. The other possibility can be the formation of chargetransfer species. It has previously been shown that the interaction between carbon nanotubes and chromophores would result in a major red-shift of optical absorption maxima for chromophores upon attachment to nanotubes through defect sites (carboxyl groups) [18]. The formation of charge transfer species between chromophores and nanotubes could also cause a red-shift of the major optical absorption, but with the appearance of low energy excitonic absorptions for the charge separated species of chromophore-attached nanotubes [19, 20]. 
Formation of charge transfer species between chromophores and nanotubes was also suggested by Raman spectroscopy. It is well known that nanotubes can form charge transfer species by electrochemical doping with redox dopants [21]. Raman spectroscopy is a very powerful tool to study these species because the intrinsic phonon line shapes of nanotubes are very sensitive to doping. Shim et al. [22] used micro-Raman spectroscopy to study the changes of intrinsic phonon line shape of nanotubes due to n-doping via noncovalent adsorption of polyethylenimine. They observed a downshift of up to $\sim 10 \mathrm{~cm}^{-1}$ of the tangential G-band spectra without line broadening for semiconducting tubes, suggesting a similar magnitude of electron transfer as commonly observed in electrochemical doping with alkali metals. Here we observed similar downshifts of G-band spectra suggesting n-doping of the nanotubes. As shown in Fig. 3, when comparing the G-band of DWNT-PEI with that of DWNT, the most intense peak at higher frequency $\left(\mathrm{G}^{+}\right)$shows downshifts of $4.4 \mathrm{~cm}^{-1}$ (from 1588.2 to $1583.8 \mathrm{~cm}^{-1}$ ) upon PEI functionalization. Even larger downshifts of the G-band are observed for DWNT-FITC $\left(10.4 \mathrm{~cm}^{-}\right.$ 1; from 1588.2 to $\left.1577.8 \mathrm{~cm}^{-1}\right)$ and DWNT-RITC $\left(11.2 \mathrm{~cm}^{-1}\right.$; from 1588.2 to $\left.1577.0 \mathrm{~cm}^{-1}\right)$, respectively. This downshift in the G-band is consistent with $\mathrm{C}-\mathrm{C}$ bond length expansion expected upon electron injection and as observed in alkali metal doping of nanotube and graphite systems [23].

The NLO properties of the various nanotubes composites were investigated using the open aperture Z-scan method, which is widely adopted as a way to investigate third-order NLO processes, including nonlinear absorption, scattering and refraction [24]. The Z-scan was carried out employing a Q-switched Nd:YAG laser of 6 ns pulses, operated at its second harmonic, 532 $\mathrm{nm}$, with a repetition rate of $10 \mathrm{~Hz}$. The laser beam was tightly focused with a $9 \mathrm{~cm}$ focus lens, 
after spatially removing higher-order modes. Meanwhile, another focusing lens was setup at $\sim 30^{\circ}$ to the direct incident beam to monitor the scattered light from the dispersions.

The mechanism leading to the optical limiting effect in carbon nanocomposites has been explored extensively $[4,5,25,26]$. Thermally induced nonlinear scattering mainly by nanotube moieties is accepted as the principle mechanism for optical limiting. In the scattering process, the high intensity beam is dispersed in order to have larger spatial dimension, hence, a reduction in the intensity is achieved. The scattering comes from the formation of scattering centers which have sizes of the order of the wavelength of the incident laser beam. The induced scattering center derives from two origins: the formation and growth of solvent bubbles, which is due to the thermal energy transfer from the carbon nanocomposites to the solvent; and the formation and expansion of carbon hybrid microplasmas, which is due to the ionization of the carbon nanohybrids. The former takes place at the lower incident energy fluences, the latter takes place at higher fluences. The extinction scattering profile follows Mie scattering theory qualitatively. In general, the optical limiting properties can be influenced by many effects, including, bundle size, solvent property, wavelength and pulse width of incident laser, etc. [4, 5]. Among them, the most intrinsic factors affecting optical limiting are the structure of the nanocomposites and the thermodynamical properties of the solvents used for dispersing them. The nanotubes composite dispersed in solvents with lower boiling points and lower surface tensions show lower limiting thresholds and better limiting effects. This is because the heat-induced solvent bubbles grow much faster in solvents with a lower liquid-gas tensile surface, thus, the bubbles can reach the critical size in a shorter time for effective scattering, resulting in a better limiting response. Furthermore, we recently verified that the appropriate solvent properties contribute to the nonlinear scattering dominated optical limiting phenomenon more than the bundle size and that 
the surface tension of the solvent plays a more important role than the viscosity or boiling point [ 27, 28 ]. On the other hand, the organic moieties of the nanocomposites make significant additional contributions to the optical limiting. The RSA from fullerene moieties and the strong linear absorption, hence saturable absorption and charge transfer from the chromophore moieties (FITC and RITC moieties), would dominate the additional contributions to the optical limiting [ 29-31].

In the NLO measurements, all of the composites were dispersed in DMF or poly(mphenylenevinylene-co-2,5-dioctoxyp-phenylenevinylene) (PmPV), followed by 20 min ultrasonic processing. PmPV is a well-known conjugated polymer used to disperse and purify carbon nanotubes, realizing property modified nanocomposites [32, 33]. The coiled polymer conformation allows it to surround layers of nanotubes, permitting sufficiently close intermolecular proximity for $\pi-\pi$ interaction to occur. The polymer has proven to be an ideal host for many types of filler and in this case it is suitable for dispersing the nanohybrids. The PmPV, dissolved in toluene, has a bright yellow color while the hybrid composites possess a deep green color, implying a strong interaction between the polymer chains and nanohybrids. Fig. 4(a) and (b) show the normalized transmission and scattered light as functions of incident energy density for DWNT-FITC and DWNT-RITC, respectively. As the energy density of incident light was increased, intense scattered light was observed from the dispersions along with decreasing transmission. Therefore the nonlinear scattering, arising from DWNT moieties, is proposed as one of the main mechanisms for optical limiting. Since the PmPV/toluene solution $(1.0 \mathrm{~g} / \mathrm{L})$ has a lower boiling point and surface tension than those of DMF, the hybrids dispersed in PmPV present a superior optical limiting response and stronger scattered signal in comparison to the DMF dispersions due to the solvent effect $[27,28]$. It should be mentioned that the contribution 
of the organic moieties of the composites to the optical limiting effect must also be counted, but the mechanism remains indeterminate.

Since the DWNT-FITC and DWNT-RITC hybrids apparently show better limiting effect in PmPV polymer dispersions than solvent DMF, we subsequently investigated the optical limiting properties of MWNT-C 60 , MWNT-FITC and MWNT-RITC dispersed in PmPV/toluene solutions. Fig. 5 shows the corresponding transmission and scattered light curves as functions of incident energy for the three MWNT hybrids. The deviations at lower incident intensity region in Fig. 5(a) and (b) are due to the experimental error in the open aperture Z-scan measurement. Table 1 summarizes the linear and nonlinear coefficients for all the tested nanocomposites. The

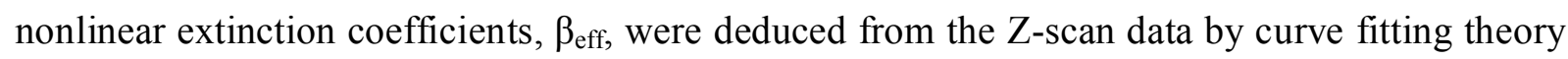
based on an intensity dependent extinction coefficient. Linear extinction coefficients, $\alpha_{0}$, as defined by $\mathrm{T}=\exp \left(-\alpha_{0} \mathrm{~L}\right)$, were measured at $532 \mathrm{~nm}$, where $\mathrm{T}$ defines the ratio of transmitted to incident laser light and $\mathrm{L}=1.0 \mathrm{~cm}$ is the sample thickness. The concentration of all nanohybrids was kept constant at $0.1 \mathrm{~g} / \mathrm{L}$. It should be noted that the linear absorption of the MWNT hybrids is much stronger than that of the DWNT hybrids at the same level of concentration of $0.1 \mathrm{~g} / \mathrm{L}$, implying the better dispersibility of MWNT hybrids in PmPV. The optical limiting response is also relatively stronger in the MWNT hybrid dispersions than in the DWNT hybrid dispersions. As addressed before, the organic moieties of the DWNT and MWNT hybrids also contribute to the optical limiting effect. We propose that, the enhanced NLO response is partly induced from the enhanced nonlinear absorption due to the RSA from fullerene moieties or the strong linear absorption, hence saturable absorption and photoinduced charge transfer from the organic chromophores moieties (donor) to nanotube moieties (acceptor). The photoinduced charge transfer followed by absorption in the charge separated excited state may enhance the NLO 
absorption, thus the NLO response. Cha et al. has reported enhanced nonlinear absorption and optical limiting in a solid film of poly(3-octylthiophene) (P3OT) sensitized with methanofullerene. The large nonlinearity is reported resulting from efficient photoinduced intermolecular charge transfer from P3OT to methanofullerene followed by absorption in the charge separated excited state [29]. The confirmation of this mechanism and the corresponding quantitative analysis has to be made by transient photoinduced absorption measurements, which is currently ongoing.

Subsequently, we studied the NLO behavior of NanoBuds, which combine fullerenes and SWNTs into a single structure in which the fullerenes are covalently bonded to the outer surface of the SWNTs, resulting in a high-efficiency pure carbon optical limiter. The covalent bonds are expected to enhance the efficiency of charge transfer between the two moieties, hence the NLO response. The SWNTs and fullerenes possess RSA and nonlinear scattering effects, respectively. We would expect a cumulative optical limiting effect from the two counterparts in one system, merging the complementary temporal and spatial NLO characteristics of them both. The NanoBuds were dispersed in N-methyl-2-pyrrolidone (NMP) and PmPV/toluene respectively for Z-scan measurements. Fig. 6(a) shows the optical limiting and optical scattering results, which indicate that the NanoBuds exhibit remarkable nonlinear scattering dominated NLO responses. In order to assess the optical limiting ability of the NanoBud dispersions, we compare the optical limiting response with that of SWNTs. As shown in Fig. 6(b), we can see that, at the same level linear transmission, the optical limiting response of NanoBud dispersions exceeds that of SWNT dispersions. It is likely that the enhanced nonlinear property of NanoBuds comes from the nonlinear absorption of $\mathrm{C}_{60}$ moiety. However, the primary mechanism dominating the optical limiting for NanoBuds is nonlinear scattering. 
To understand the NLO process for the NanoBuds as well as DWNT- $\mathrm{C}_{60}$ and MWNT-C 60 , we give a brief description of their nonlinear optical process. Within the ns pulse excitation duration, fullerenes dominate the nonlinear optical effect in the first picoseconds regime due to the rapid response of RSA. As a consequence of thermal energy transfer from nanotubes to the solvent, the nonlinear scattering then dominates the nonlinear process when the solvent and/or carbon vapor bubbles reach the critical size. Meanwhile, the charge transfer may contribute to the NLO response of the nanohybrids as well. It should be mentioned that the optimization of the optical limiting performance of the three fullerene-related nanohybrids can potentially be achieved by choosing the nanohybrids with an appropriate fullerene density. The cumulative optical limiting effect happens when the two moieties have comparable optical limiting responses. If one moiety dominates, the whole optical limiting performance will be close to that of the moiety [7].

\section{Summary}

The NLO properties of DWNT hybrids, MWNT hybrids and NanoBuds were investigated in PmPV/toluene and DMF dispersions using Z-scan method. These nanotube composites show remarkable optical limiting performance, due to a merged effect of the complementary NLO characteristics of the moieties of the composites. Nonlinear scattering was confirmed as the main mechanism for the optical limiting. RSA makes a significant contribution to the NLO response of MWNT- $\mathrm{C}_{60}$ and NanoBuds. Photoinduced charge transfer from the FITC and RITC to nanotubes may enhance the nonlinear absorption, thus an effective optical limiting response. 


\section{Acknowledgments}

This work was supported in part by the US Department of Energy under Grant No. DE-FG3608GO88008. J.W. thanks the financial supports from the 100-Talent Program of Chinese Academy of Sciences, the starting grant of Shanghai Institute of Optics and Fine Mechanics (1108221-JR0), the National Natural Science Foundation of China (NSFC, Grant No. 61178007), and Science and Technology Commission of Shanghai Municipality (STCSM Nano Project, Grant No. 11nm0502400). L.Z. thanks the financial supports from NSFC (Grant No. 50802103 and 51072207), STCSM Excellent Academic Leader of Shanghai (Grant No. 10XD1404600) and the Shanghai Natural Science Foundation (Grant No. 11ZR1441500). J.W. acknowledges Mr. Darren Lee for his helpful discussion in this work. 


\section{References}

[1] L.W. Tutt, T.F. Boggess, A review of optical limiting mechanisms and devices using organics, fullerenes, semiconductors and other materials, Progress in Quantum Electronics, 17 (1993) 299-338.

[2] Y.P. Sun, J.E. Riggs, Organic and inorganic optical limiting materials. From fullerenes to nanoparticles, International Reviews in Physical Chemistry, 18 (1999) 43-90.

[3] Y. Chen, Y. Lin, Y. Liu, J. Doyle, N. He, X.D. Zhuang, J.R. Bai, W.J. Blau, Carbon nanotube-based functional materials for optical limiting, Journal of Nanoscience and Nanotechnology, 7 (2007) 1268-1283.

[4] J. Wang, W.J. Blau, Inorganic and Hybrid Nanostructures for Optical Limiting, Journal of Optics A - Pure and Applied Optics, 11 (2009) 024001.

[5] J. Wang, Y. Chen, W.J. Blau, Carbon Nanotubes and Nanotube Composites for Nonlinear Optical Devices, Journal of Materials Chemistry, 19 (2009) 7425-7443.

[6] W. Blau, H. Byrne, W.M. Dennis, J.M. Kelly, Reverse saturable absorption in tetraphenylporphyrins, Optics Communications, 56 (1985) 25-29.

[7] N. Izard, C. Menard, D. Riehl, E. Doris, C. Mioskowski, E. Anglaret, Combination of carbon nanotubes and two-photon absorbers for broadband optical limiting, Chemical Physics Letters, 391 (2004) 124-128.

[8] S. Webster, M. Reyes-Reyes, X. Pedron, R. López-Sandoval, M. Terrones, D.L. Carroll, Enhanced nonlinear transmittance by complementary nonlinear mechanisms: a reverse-saturable absorbing dye blended with nonlinear-scattering carbon nanotubes, Advanced Materials, 17 (2005) 1239-1243. 
[9] Z.B. Liu, J.G. Tian, Z. Guo, D.M. Ren, F. Du, J.Y. Zheng, Y.S. Chen, Enhanced Optical Limiting Effects in Porphyrin-Covalently Functionalized Single-Walled Carbon Nanotubes, Advanced Materials, 20 (2008) 511-515.

[10] J. Wang, W.J. Blau, Linear and nonlinear spectroscopic studies of phthalocyanine-carbon nanotube blends, Chemical Physics Letters, 465 (2008) 265-271.

[11] N. He, Y. Chen, J. Bai, J. Wang, W.J. Blau, J. Zhu, Preparation and Optical Limiting Properties of Multiwalled Carbon Nanotubes with $\pi$-Conjugated Metal-Free Phthalocyanine Moieties, Journal of Physical Chemistry C, 113 (2009) 13029-13035.

[12] K.-S. Liao, J. Wang, D. Früchtl, N.J. Alley, E. Andreoli, E.P. Dillon, A.R. Barron, H. Kim, H.J. Byrne, W.J. Blau, S.A. Curran, Optical limiting study of double wall carbon nanotubefullerene hybrids, Chemical Physics Letters, 489 (2010) 207-211.

[13] A.G. Nasibulin, P.V. Pikhitsa, H. Jiang, D.P. Brown, A.V. Krasheninnikov, A.S. Anisimov, P. Queipo, A. Moisala, D. Gonzalez, G. Lientschnig, A. Hassanien, S.D. Shandakov, G. Lolli, D.E. Resasco, M. Choi, D. Tomanek, E.I. Kauppinen, A novel hybrid carbon material, Nat Nano, $2(2007) 156-161$.

[14] T. Hasan, Z. Sun, A.C. Ferrari, Nanotube-polymer composites for ultrafast photonics, Advance Materials, 21 (2009) 3874-3899.

[15] F. Bonaccorso, Z. Sun, T. Hasan, A.C. Ferrari, Graphene photonics and optoelectronics, Nature Photonics, 4 (2010) 611-622.

[16] A.G. Nasibulin, A.S. Anisimov, P.V. Pikhitsa, H. Jiang, D.P. Brown, M. Choi, E.I. Kauppinen, Investigations of NanoBud formation, Chemical Physics Letters, 446 (2007) 109114. 
[17] K.-S. Liao, A. Wan, J.D. Batteas, D.E. Bergbreiter, Superhydrophobic Surfaces Formed Using Layer-by-Layer Self-Assembly with Aminated Multiwall Carbon Nanotubes, Langmuir, 24 (2008) 4245-4253.

[18] K.A.S. Fernando, Y. Lin, W. Wang, S. Kumar, B. Zhou, S.-Y. Xie, L.T. Cureton, Y.-P. Sun, Diminished Band-Gap Transitions of Single-Walled Carbon Nanotubes in Complexation with Aromatic Molecules, Journal of the American Chemical Society, 126 (2004) 10234-10235.

[19] S. Campidelli, B. Ballesteros, A. Filoramo, D. Diaz Diaz, G. de la Torre, T.s. Torres, G.M.A. Rahman, C. Ehli, D. Kiessling, F. Werner, V. Sgobba, D.M. Guldi, C. Cioffi, M. Prato, J.-P. Bourgoin, Facile Decoration of Functionalized Single-Wall Carbon Nanotubes with Phthalocyanines via Click Chemistry, Journal of the American Chemical Society, 130 (2008) 11503-11509.

[20] J. Yu, S. Mathew, B.S. Flavel, M.R. Johnston, J.G. Shapter, Ruthenium Porphyrin Functionalized Single-Walled Carbon Nanotube Arrays - Step Toward Light Harvesting Antenna and Multibit Information Storage, Journal of the American Chemical Society, 130 (2008) 87888796.

[21] L. Kavan, L. Dunsch, Spectroelectrochemistry of Carbon Nanostructures, ChemPhysChem, 8 (2007) 974-998.

[22] M. Shim, T. Ozel, A. Gaur, C. Wang, Insights on Charge Transfer Doping and Intrinsic Phonon Line Shape of Carbon Nanotubes by Simple Polymer Adsorption, Journal of the American Chemical Society, 128 (2006) 7522-7530.

[23] G. Chen, C.A. Furtado, U.J. Kim, P.C. Eklund, Alkali-metal-doping dynamics and anomalous lattice contraction of individual debundled carbon nanotubes, Physical Review B, 72 (2005) 155406. 
[24] M. Sheikbahae, A.A. Said, T.H. Wei, D.J. Hagan, E.W. Vanstryland, Sensitive Measurement of Optical Nonlinearities Using a Single Beam, IEEE Journal of Quantum Electronics, 26 (1990) 760-769.

[25] P. Chen, X. Wu, X. Sun, J. Lin, W. Ji, K.L. Tan, Electronic structure and optical limiting behavior of carbon nanotubes, Physical Review Letters, 82 (1999) 2548-2551.

[26] L. Vivien, P. Lancon, D. Riehl, F. Hache, E. Anglaret, Carbon nanotubes for optical limiting, Carbon, 40 (2002) 1789-1797.

[27] J. Wang, D. Früchtl, W.J. Blau, The importance of solvent properties for optical limiting of carbon nanotube dispersions, Optics Communications, 283 (2010) 464-468.

[28] J. Wang, D. Früchtl, Z. Sun, J.N. Coleman, W.J. Blau, Control of Optical Limiting of Carbon Nanotube Dispersions by Changing Solvent Parameters, The Journal of Physical Chemistry C, 114 (2010) 6148-6156.

[29] M. Cha, N.S. Sariciftci, A.J. Heeger, J.C. Hummelen, F. Wudl, Enhanced nonlinear absorption and optical limiting in semiconducting polymer/methanofullerene charge transfer films, Applied Physics Letters, 67 (1995) 3850.

[30] R. Chitta, A.S.D. Sandanayaka, A.L. Schumacher, L. D’Souza, Y. Araki, O. Ito, F. D’Souza, Donor-acceptor nanohybrids of zinc naphthalocyanine or zinc porphyrin noncovalently linked to single-wall carbon nanotubes for photoinduced electron transfer, Journal of Physical Chemistry C, 111 (2007) 6947-6955.

[31] N.K.M.N. Srinivas, S.V. Rao, D.N. Rao, Saturable and reverse saturable absorption of Rhodamine B in methanol and water, J. Opt. Soc. Am. B, 20 (2003) 2470-2479.

[32] S.A. Curran, P.M. Ajayan, W.J. Blau, D.L. Carroll, J.N. Coleman, A.B. Dalton, A.P. Davey, A. Drury, B. McCarthy, S. Maier, A. Strevens, A composite from poly(m-phenylenevinylene-co- 
2,5-dioctoxy-p-phenylenevinylene) and carbon nanotubes: A novel material for molecular optoelectronics, Advanced Materials, 10 (1998) 1091-1093.

[33] S. Curran, A.P. Davey, J. Coleman, A. Dalton, B. McCarthy, S. Maier, A. Drury, D. Gray, M. Brennan, K. Ryder, M. Lamy de la Chapelle, C. Joumet, P. Bemier, H.J. Byme, D. Carroll, P.M. Ajayan, S.J. Lefrant, W.J. Blau, Evolution and evaluation of the polymer/nanotube composite, Synthetic Metals, 103 (1999) 2559-2562. 
Table 1. Linear and NLO coefficients for all the tested nanohybrids. The concentration of the dispersions was $0.1 \mathrm{~g} / \mathrm{L}$ except for the NanoBud and SWNT dispersions.

\begin{tabular}{|c|c|c|c|c|}
\hline Materials & Hosts & $\mathrm{T}[\%]$ & $\alpha_{0}\left[\mathrm{~cm}^{-1}\right]$ & $\beta_{\text {eff }}\left[\mathrm{cm} \mathrm{GW}^{-1}\right]$ \\
\hline & Chlorobenzene & 79.8 & 0.23 & $7.01 \pm 0.99$ \\
\hline \multicolumn{5}{|l|}{ DWNT-C $60[12]$} \\
\hline & PmPV/toluene & 70.9 & 0.34 & $6.02 \pm 1.03$ \\
\hline & DMF & 82.1 & 0.20 & $2.01 \pm 0.38$ \\
\hline \multicolumn{5}{|l|}{ DWNT-FITC } \\
\hline & PmPV/toluene & 85.1 & 0.16 & $4.69 \pm 1.30$ \\
\hline & DMF & 79.9 & 0.22 & $1.55 \pm 0.37$ \\
\hline \multicolumn{5}{|l|}{ DWNT-RITC } \\
\hline & PmPV/toluene & 85.5 & 0.16 & $3.44 \pm 0.50$ \\
\hline MWNT-C 60 & PmPV/toluene & 40.8 & 0.90 & $6.64 \pm 0.72$ \\
\hline MWNT-FITC & PmPV/toluene & 35.2 & 1.04 & $7.70 \pm 1.15$ \\
\hline MWNT-RITC & $\mathrm{PmPV} /$ toluene & 42.4 & 0.86 & $7.98 \pm 1.58$ \\
\hline & $\mathrm{PmPV} /$ toluene & 50.7 & 0.68 & $2.23 \pm 0.21$ \\
\hline \multicolumn{5}{|l|}{ NanoBuds } \\
\hline & NMP & 54.4 & 0.61 & $2.71 \pm 0.38$ \\
\hline SWNTs & NMP & 54.2 & 0.61 & $1.96 \pm 0.23$ \\
\hline
\end{tabular}




\section{Figure captions}

Fig. 1. Syntheses of DWNT-FITC and DWNT-RITC.

Fig. 2. UV-vis spectra of DWNT-FITC and DWNT-RITC in water. Spectra are offset for clarity.

Fig. 3. Raman spectra of DWNT, DWNT-PEI, DWNT-FITC and DWNT-RITC. Intensities of all spectra are normalized with respect to the G-mode of DWNT and all spectra are offset for clarity.

Fig. 4. Nonlinear transmission and nonlinear scattering as functions of incident energy density for (a) DWNT-FITC and (b) DWNT-RITC.

Fig. 5. Nonlinear transmission and nonlinear scattering as functions of incident energy density for (a) MWNT-C 60 , (b) MWNT-FITC and (c) MWNT-RITC.

Fig. 6. (a) Nonlinear transmission and nonlinear scattering as functions of incident energy density for NanoBuds in NMP and PmPV/toluene; (b) Optical limiting performance of NanoBuds and SWNTs at the same level of linear transmission. 


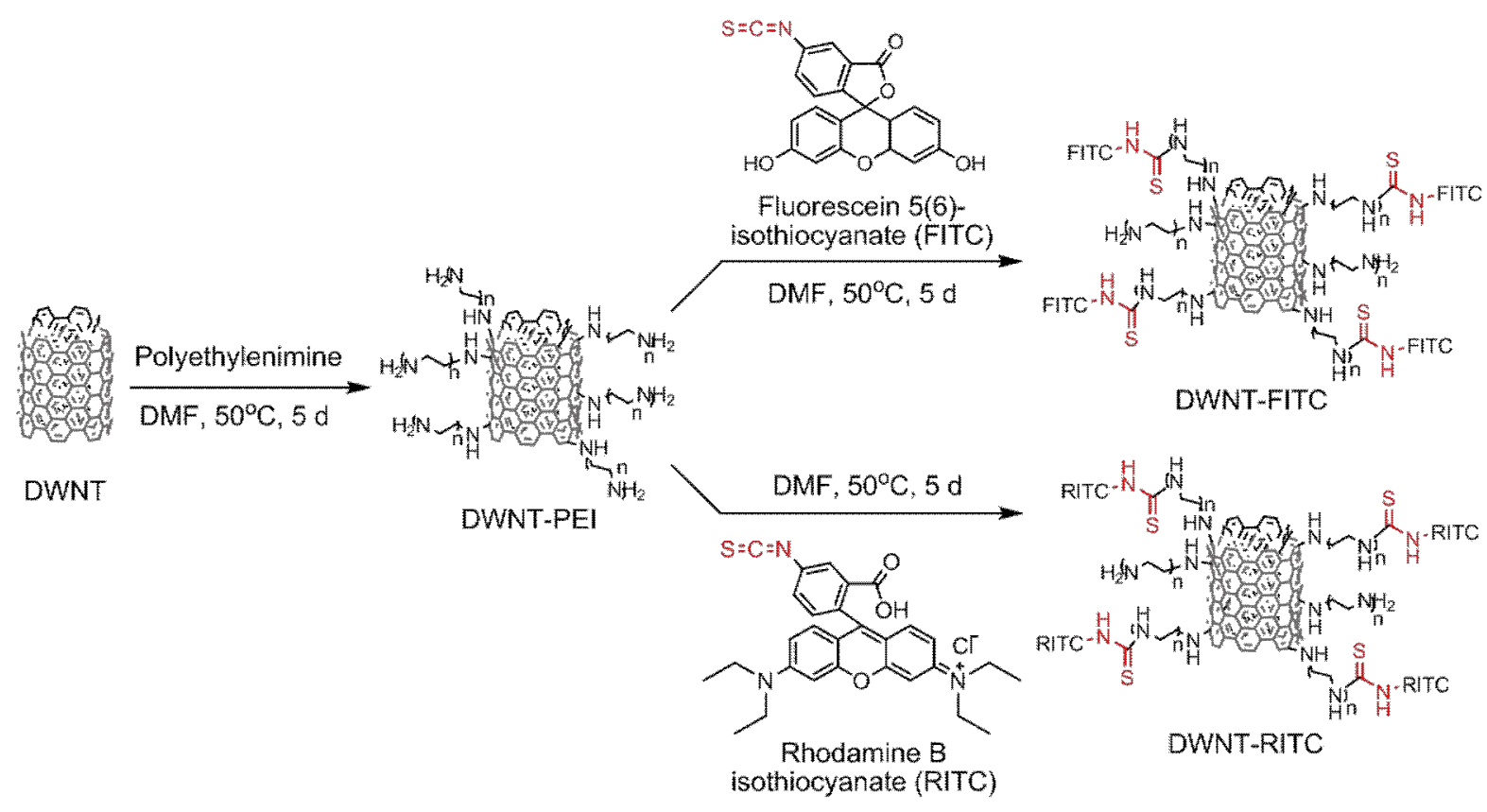

Fig. 1 

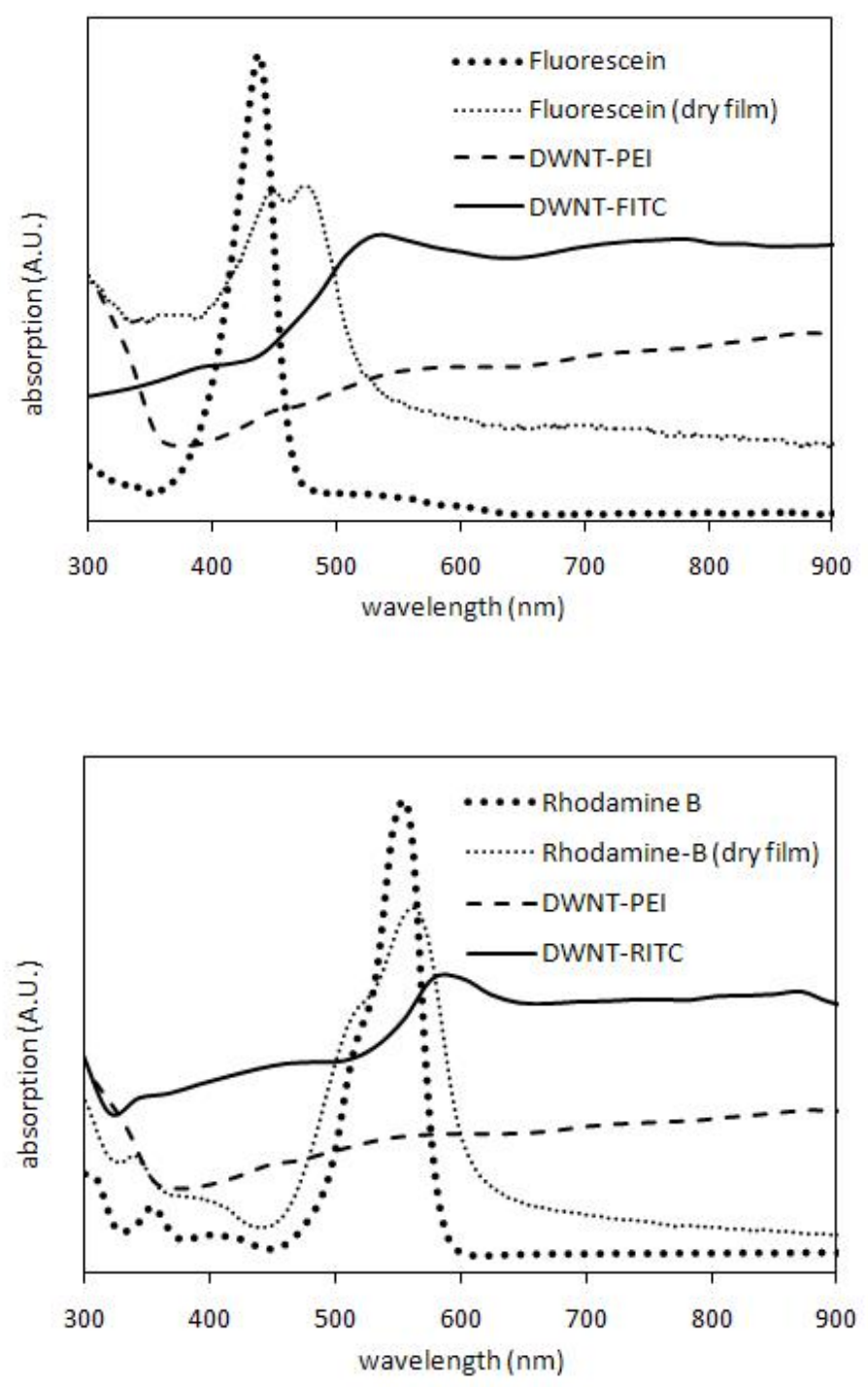

Fig. 2 


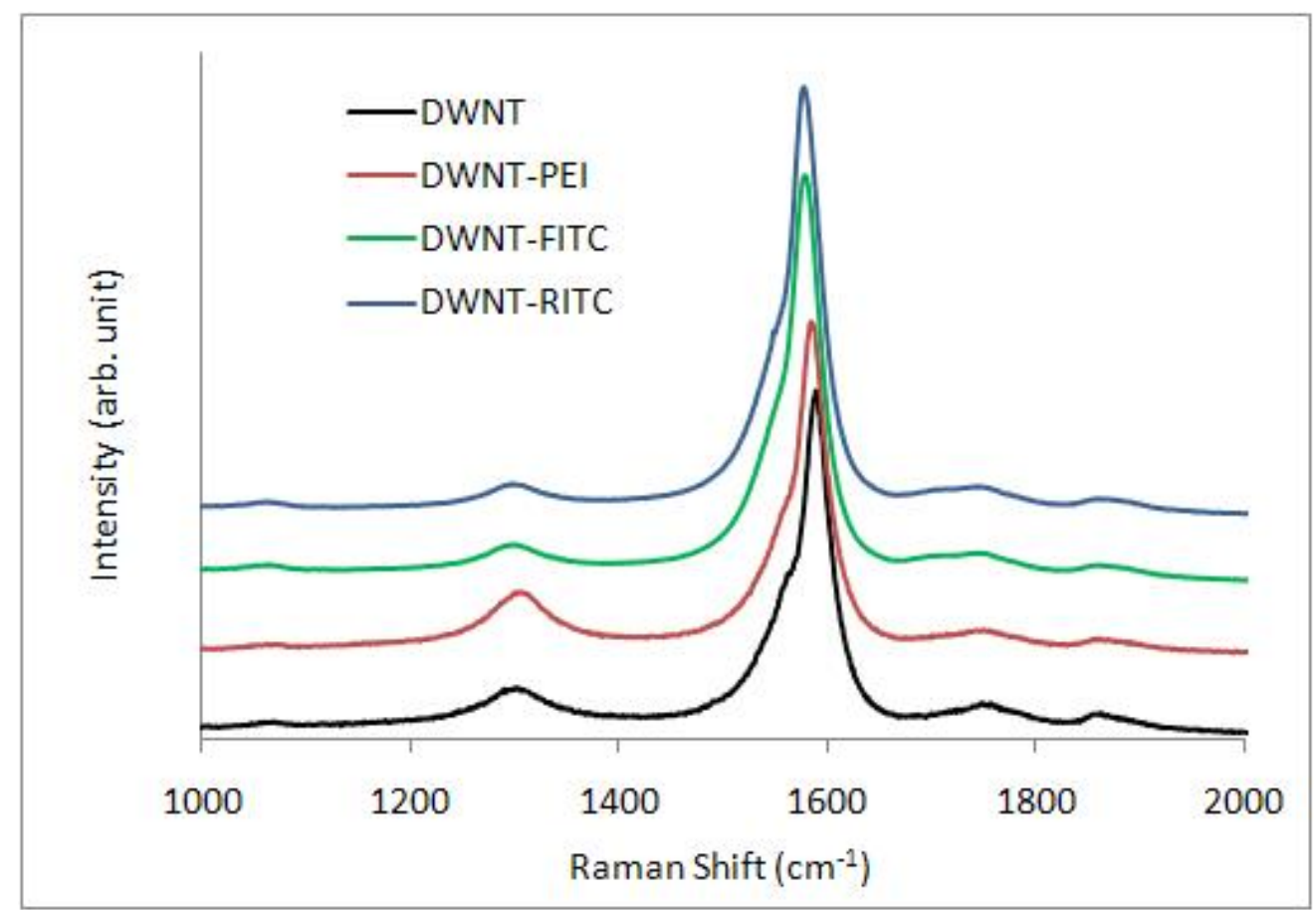

Fig. 3 


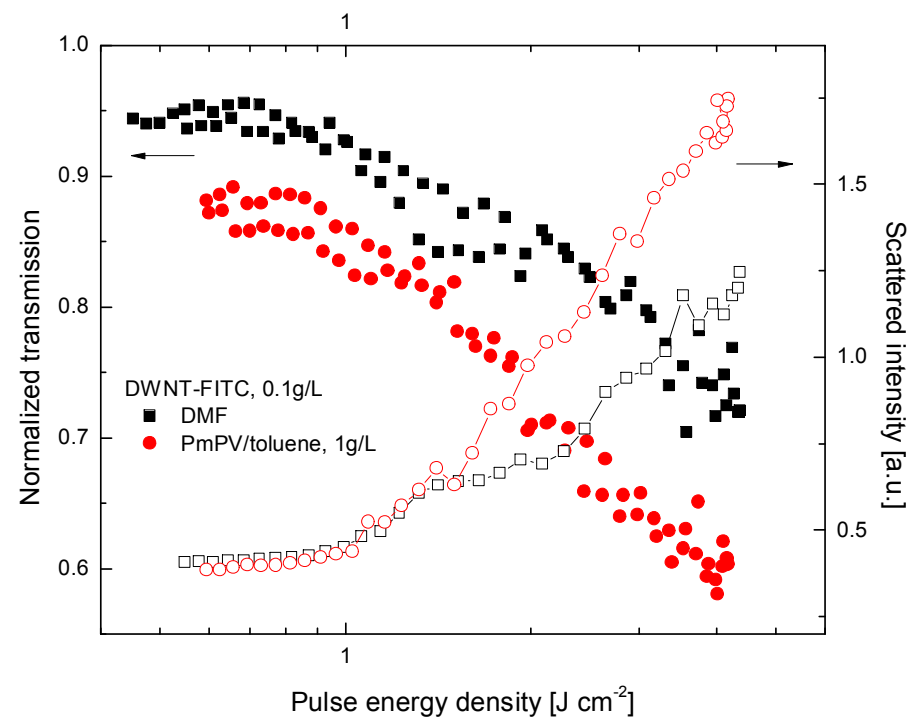

(a)

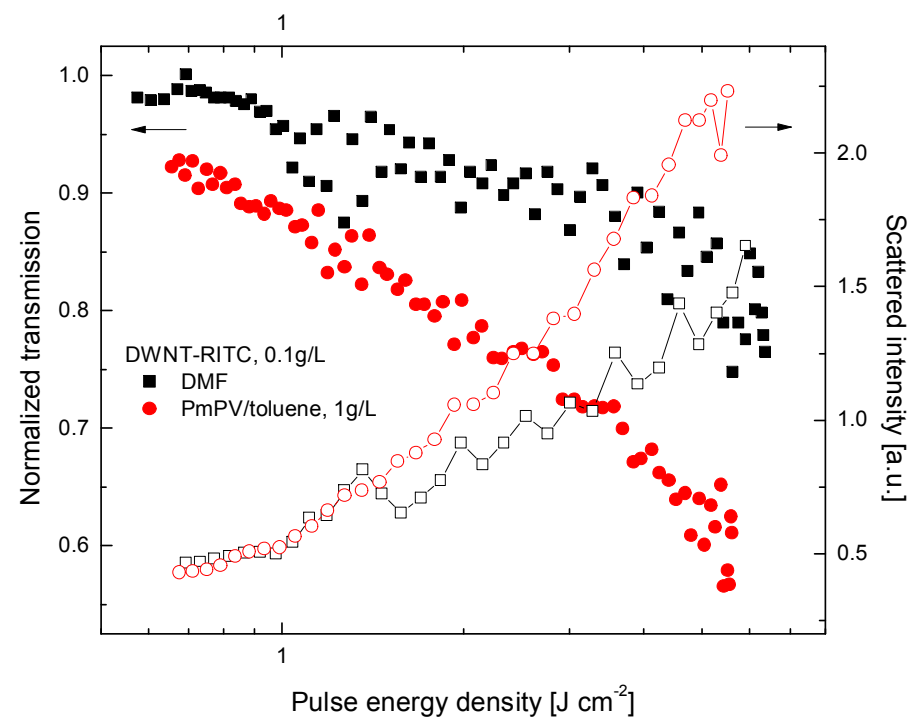

(b)

Fig. 4 


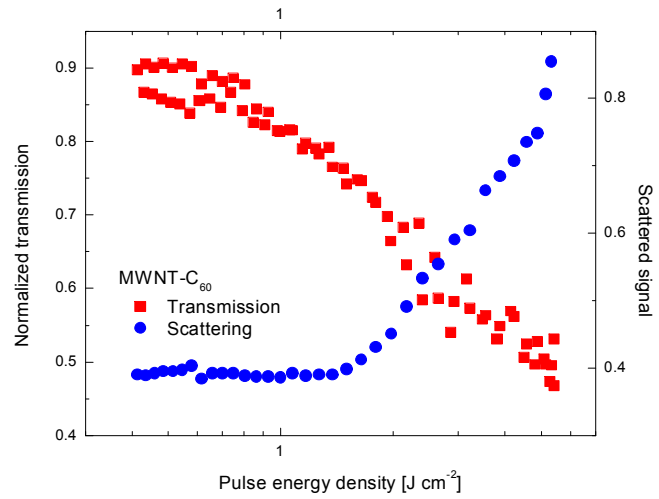

(a)

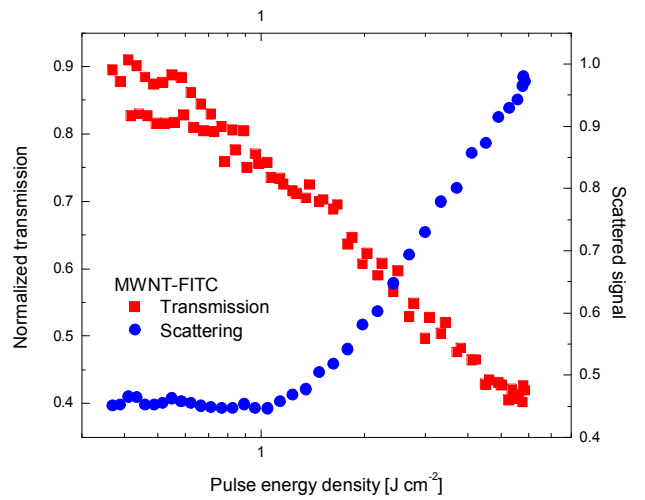

(b)

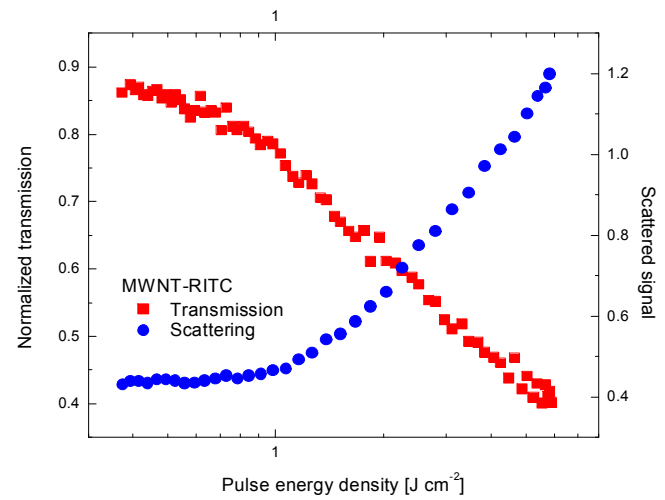

(c)

Fig. 5 


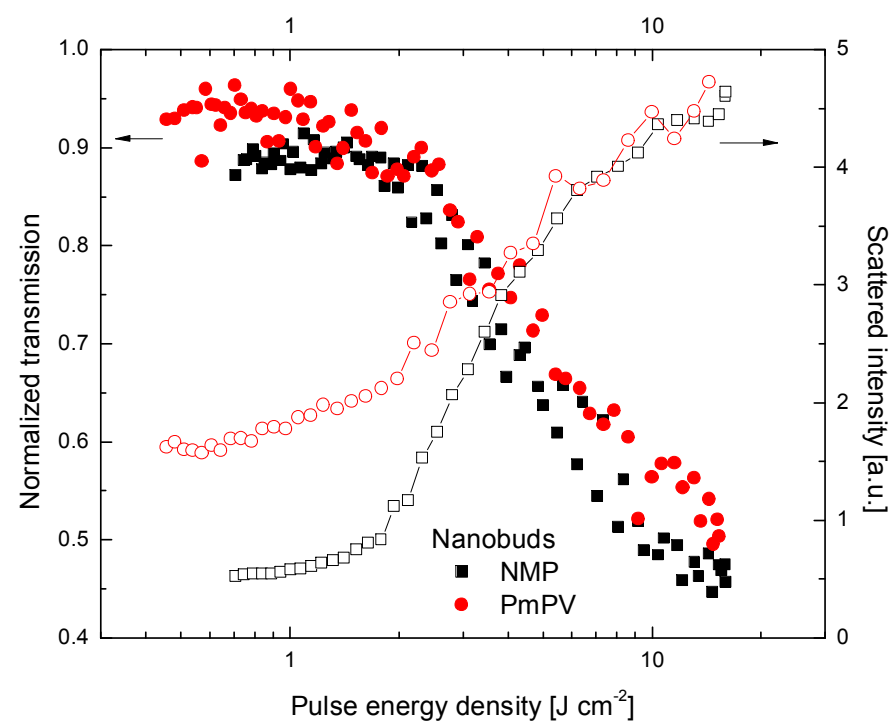

(a)

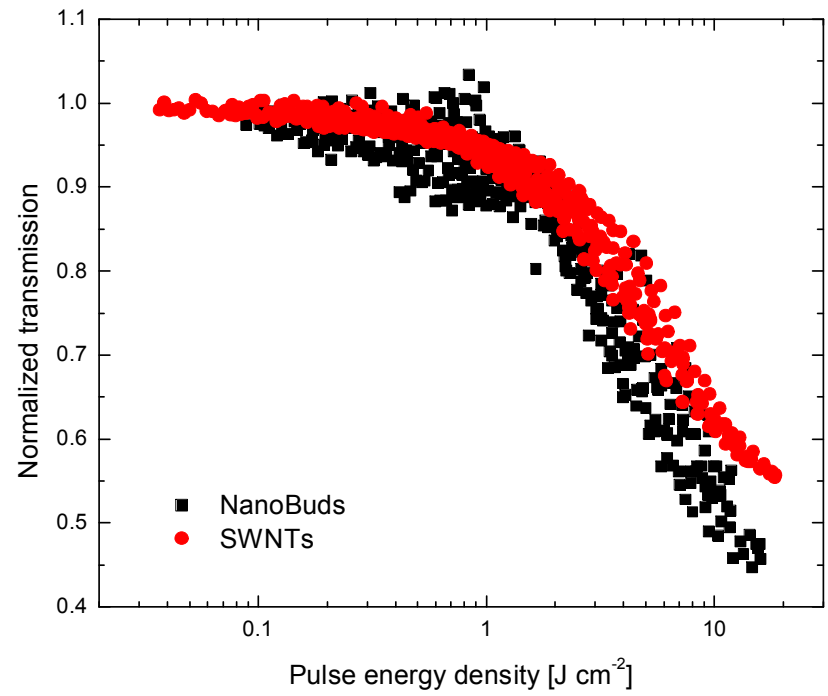

(b)

Fig. 6 
Ref. MATCHEMPHYS-D-11-02353

Title: Nonlinear Optical Properties of Carbon Nanotube Hybrids in Polymer Dispersions

Corresponding Author: Prof. Jun Wang

\section{Declaration}

I declare that there is no conflict of interest exists in the article. If accepted, the article will not be published elsewhere in the same form, in any language, without the written consent of the publisher.

Jun Wang

Prof. Jun Wang, Ph.D

Key Laboratory of Materials for High-Power Laser

Shanghai Institute of Optics and Fine Mechanics,

Chinese Academy of Sciences, Shanghai 201800, China 